\title{
Applications, results and future direction (EAIS 12)
}

\author{
José Antonio Iglesias • Igor Škrjanc
}

Published online: 8 February 2014

(c) Springer-Verlag Berlin Heidelberg 2014

Nowadays, one of the main characteristics of the intelligent system is their level of autonomy. In order to provide these systems with a high level of both autonomy and flexibility, new methods, algorithms and implementations of systems are emerging.

The environment in which these systems operate is usually non-stationary, very (and unpredictably) changeable and even unknown. Thus, an intelligent system needs to be able to take into account these changes by changing their behavior, adapting their structure, self-evaluating and self-developing. These actions are needed in order to achieve the goal of evolving the whole system with environment. This is the main idea behind the emerging area of Evolving and Adaptive Intelligent Systems.

The goal of the Evolving and Adaptive Intelligent Systems is the development of on-line learning methods and computationally efficient algorithms whose structure is very flexible in order to adapt to the environment. There are many different areas, in which these systems are being used such as assisted ambient intelligence, intelligent systems, robotics, environmental monitoring, or security.

This special issue includes 6 selected and carefully peer reviewed papers which are extended versions of papers submitted to the 2012 IEEE Evolving and Adaptive

\section{J. A. Iglesias ( $\square)$}

Carlos III University of Madrid, Madrid, Spain

e-mail: jiglesia@inf.uc3m.es

URL: http://www.caos.inf.uc3m.es/ jiglesia/

\section{I. Škrjanc}

Faculty of Electrical Engineering, University of Ljubljana,

Ljubljana, Slovenia

e-mail: igor.skrjanc@fe.uni-lj.si

URL: http://msc.fe.uni-lj.si
Intelligent Systems Conference, (EAIS 12) which was held in Madrid, Spain.

In the first paper "A robust fuzzy adaptive law for evolving control systems" by Sašo Blažič, Igor Škrjanc and Drago Matko an adaptive law with leakage is presented. The law can be used in the consequent part of Takagi-Sugeno-based control. The approach enables easy implementation in the control systems with evolving antecedent part. This combination results in a high-performance and robust control of nonlinear and slowly varying systems. The proposed adaptive law is a natural way to cope with the parasitic dynamics. Moreover, the value of the leakage parameter sigma' is directly related to the norm of the parasitic dynamics. The boundedness of estimated parameters, the tracking error and all the signals in the system is proven in certain condition. This means that the proposed adaptive law ensures the global stability of the system.

The second paper "Towards the online learning with Kernels in classification and regression" by Guoqi Li, Guangshe Zhao and Feng Yang, solves a constrained optimization model by using optimization models and algorithms for online learning with kernels in classification and regression in a RKHS (Kernel Hilbert Space). The authors propose a new optimization model and derive new algorithms which are tested in a few examples in classification and regression. This proposal strengths the foundation of online learning with kernels and also enriches the research area of SVM.

The third paper "Fault-tolerant gait learning and morphology optimization of a polymorphic walking robot" by David Johan Christensen, Jørgen Christian Larsen and Kasper Stoy, presents a control strategy for online life-long learning of locomotion gaits. The experimental platform is a quadruped robot assembled from the LocoKit modular 
robotic construction kit. The learning strategy proposed in this paper applies a stochastic optimization algorithm to optimize eight open parameters of a central pattern generator based gait implementation. The experiments in this paper were performed on continued adaptation after failures of several actuators and the authors found that the system was able to readapt after such failures.

Massimiliano Dal Mas is the author of the fourth paper titled "Elastic Adaptive Dynamics Methodology on Ontology Matching on Evolving Folksonomy Driven Environment". This paper considers ontologies based on folksonomies according to a new concept structure called "Folksodriven" to represent folksonomies. Folksonomies are a set of terms that a group of users tagged content without a controlled vocabulary. In this paper, a Folksodriven Structure Network (FSN), built from the relations among the Folksodriven tags (FD tags), is presented as a folksonomy tags suggestions for the user to solve the problems inherent in an uncontrolled vocabulary of the folksonomy. As it is explained in this paper, the proposed study of the elasticity for the FSN promotes the development of defect theories for the plasticity and elasto-adaptative-dynamics.

The fifth paper "Dynamic learning in cognitive robotics through a procedural long term memory" by Francisco Bellas, Pilar Caamaño, Andrés Faiña and Richard J. Duro shows the advantages of using a Long-Term Memory (LTM) for optimizing the adaptive learning capabilities of a cognitive robot in dynamic environments. Specifically, a procedural LTM that stores basic models and behaviours is included in the evolutionary-based Multilevel Darwinist Brain (MDB) cognitive architecture. The authors demonstrate in the paper the advantage of having this type of memory element to optimize the adaptive learning capabilities of the robot and, consequently, its autonomy level.

The sixth paper "Adaptive evolving strategy for dextrous robotic manipulation" by César Arismendi, David Álvarez, Santiago Garrido, Luis Moreno proposes an algorithm for adapting manipulation trajectories for different locations. The adaptation is achieved by optimizing in position, orientation and energy consumption. The approach is built over the basis of Evolution Strategies, and only uses forward kinematics permitting to avoid all the inconveniences that inverse kinematics imply, as well as convergence problems in singular kinematic configurations. Manipulation paths generated with this algorithm can achieve optimal performance, sometimes even improving original path smoothness. The authors present experimental tests that show the ability to apply the algorithm in real-time for obtaining adapted manipulation paths, proving to be a feasible solution for mobile robots manipulation problems.

Finally, the guest editors would like to thank all the authors and reviewers who contributed to this special issue. Also, we wish to thank the editors-in-chief and editorial office of the Evolving Systems Journal for their support. 